\title{
ORIGINAL RESEARCH \\ Measuring Myelin Repair and Axonal Loss with Diffusion Tensor Imaging
}

R.J. Fox

T. Cronin

J. Lin

X. Wang

K. Sakaie

D. Ontaneda

S.Y. Mahmoud

M.J. Lowe

M.D. Phillips
BACKGROUND AND PURPOSE: DTI is an MR imaging measure of brain tissue integrity and provides an attractive metric for use in neuroprotection clinical trials. The purpose of our study was to use DTI to evaluate the longitudinal changes in brain tissue integrity in a group of patients with MS.

MATERIALS AND METHODS: Twenty-one patients with MS starting natalizumab were imaged serially for 12 months. Gadolinium-enhancing lesions and 20 regions of interest from normal-appearing white and gray matter brain tissue were followed longitudinally. Average values within each region of interest were derived for FA, $\lambda_{\|}, \lambda_{\perp}$, and MD. New T1 black holes were identified at 12 months. Analysis was performed by using mixed-model regression analysis with slope (ie, DTI change per month) as the dependent variable.

RESULTS: During 1 year, FA increased in gadolinium-enhancing lesions but decreased in NABT $(P<$ .0001 for both). Changes in FA within gadolinium-enhancing lesions were driven by decreased $\lambda_{\perp}(P<$ .001), and within NABT, by decreased $\lambda_{\|}(P<.0001)$. A higher $\lambda_{\perp}$ within gadolinium-enhancing lesions at baseline predicted conversion to T1 black holes at 12 months. MD was unchanged in both gadolinium-enhancing lesions and NABT.

CONCLUSIONS: We observed changes in DTI measures during 1 year in both gadolinium-enhancing lesions and NABT. The DTI results may represent possible remyelination within acute lesions and chronic axonal degeneration in NAWM. These results support the use of DTI as a measure of tissue integrity for studies of neuroprotective therapies.
M $S$ is a chronic demyelinating disease of the central nervous system. Conventional MR imaging readily identifies the classic hallmarks of MS in white matter yet has distinct disadvantages. ${ }^{1}$ Focal lesions identify areas of active inflammation and tissue injury but do not characterize the degree of tissue injury. Despite an absence of new lesions in patients with progressive MS, chronic progressive disability is observed for many years. Altogether, conventional imaging is a poor measure of the degree of acute injury, later recovery, and chronic degeneration. More advanced imaging modalities are needed to characterize the degree of tissue injury and its response to therapies more accurately.

Received March 15, 2010; accepted after revision May 31.

From the Mellen Center (R.J.F., T.C., D.O.), Department of Neurology; Department of Radiology (J.L., K.S., S.Y.M., M.J.L., M.D.P.), Neurological Institute; and Department of Quantitative Health Sciences (X.W.), Cleveland Clinic Foundation, Cleveland, Ohio.

The corresponding author takes full responsibility for the data, analysis, and interpretation and had full access to all data. Statistical analysis was performed by coauthor Dr Xiaofeng Wang.

This work was supported by the National Institutes of Health (K23NS47211), National Multiple Sclerosis Society (RG 3548A2), and Nancy Davis Center Without Walls.

Paper previously presented in part at: Annual Meeting of the American Society of Neuroradiology, May 16-21, 2009; Vancouver, British Columbia, Canada; and the American Academy of Neurology, April 25-May 2, 2009; Seattle, Washington.

Please address correspondence to Robert J. Fox, MD, Mellen Center for Multiple Sclerosis Treatment and Research, Cleveland Clinic Foundation, 9500 Euclid Ave, U-10, Cleveland, OH 44195; e-mail: foxr@ccf.org

Indicates open access to non-subscribers at www.ajnr.org

DOI 10.3174/ajnr.A2238
DTI is a quantitative MR imaging technique that measures the $3 \mathrm{D}$ diffusion of water molecules within tissue through the application of multiple diffusion gradients. ${ }^{2}$ The power of diffusion techniques comes from measurement of molecular probing of tissue structures by water molecules at a microscopic scale well beyond the typical MR imaging resolution. By measuring the interaction of water molecules with cell membranes, myelin sheaths, and macromolecules, tissue integrity can be inferred. ${ }^{3}$ Tissue has physical structures that limit diffusion in different directions, so diffusion is typically described as a $3 \mathrm{D}$ ellipsoid through a $3 \times 3$ matrix. In nervous tissue, the largest (or primary) ellipsoid eigenvector represents diffusion along the length of a fiber tract and so is referred to as the $\lambda_{\|}$. The other shorter 2 ellipsoid eigenvectors, when averaged together, are called $\lambda_{\perp}$. Clinical and pathologic studies have found that $\lambda_{\|}$tends to reflect axonal integrity, whereas $\lambda_{\perp}$ corresponds more closely to myelin integrity. ${ }^{4-9}$ Animal and human studies suggest that $\lambda_{\|}$and $\lambda_{\perp}$ are approximate measures of axonal and myelin integrity, particularly in acute injury.

Diffusion ellipsoids in highly organized fiber tracts (eg, pyramidal tracts and the corpus callosum) are very elongated. The absence of fiber tracts in gray matter makes diffusion ellipsoids less elongated, though gray matter still demonstrates some anisotropy. FA is a common metric to describe the degree of diffusion directionality or elongation. A high FA within a single voxel would signify that diffusion occurs predominantly along a single axis. A low FA would signify that diffusion occurs along all 3 cardinal axes. An overall measure 
of diffusion magnitude is described by MD, which ignores anisotropy and simply describes the overall magnitude of diffusion.

Given the limited dynamic range of conventional imaging to characterize tissue integrity, DTI is an attractive alternative to conventional imaging for measuring tissue integrity in longitudinal MS studies and the potential impact of therapy. Although several studies have correlated DTI measures with functional performance, ${ }^{10-12}$ little is known about how DTI measures tissue recovery and later degeneration. Similarly, to our knowledge, few studies have evaluated the changes in brain tissue during treatment with natalizumab, and no studies have evaluated patients with MS after starting immunomodulating therapy. In this study, we evaluated the longitudinal change in DTI measures within focal brain regions of patients with MS starting highly effective anti-inflammatory therapy. Specifically, we evaluated the ability of DTI of the brain to characterize changes following acute inflammatory injury and chronic changes in NABT. We hypothesized that longitudinal DTI imaging will measure changes in brain tissue integrity in both gadolinium-enhancing lesions and NABT.

\section{Materials and Methods}

\section{Participants}

After obtaining local institutional review board approval, we enrolled 22 patients with MS into a longitudinal observational DTI study starting treatment with natalizumab, with baseline MR imaging immediately before the first natalizumab infusion. Patients were enrolled sequentially into this natural history study from an academic specialty clinical center. Inclusion criteria were clinically definite MS by the Revised McDonald Diagnostic Criteria, age $>18$ years, and ability to provide informed consent. Exclusion criteria were clinical relapse or steroid treatment in the previous 8 weeks (to minimize the potential confounding of corticosteroids), pregnancy, and contraindications to MR imaging, such as severe claustrophobia and implanted devices, such as neurostimulators, pacemakers, and so forth. Patients with MS started natalizumab for clinical indications, according to the US Food and Drug Administration label. Because this was a small pilot study funded by the National Institutes of Health and National Multiple Sclerosis Society, no untreated controls were studied. Written informed consent was obtained from all participants. One patient discontinued imaging follow-up after only 2 months and was removed from the data analysis.

\section{MR Imaging Protocol}

Imaging was performed on a 3T Trio scanner (Siemens Medical Systems, Erlangen, Germany). Diffusion-weighted imaging used 71 noncollinear diffusion-weighting gradients $(2.5 \times 2.5 \times 2.5 \mathrm{~mm}$ voxels, $b=2000 \mathrm{~s} / \mathrm{mm}^{2}, 8 b=0$ acquisitions, $256 \times 256 \mathrm{~mm}$ FOV, $102 \times 102$ matrix, forty-eight $2.5-\mathrm{mm}$ thick sections, TE/TR $=95 / 7300 \mathrm{~ms}$ ). Anatomic imaging was performed for lesion detection and coregistration: 3D MPRAGE $(256 \times 256 \mathrm{~mm}$ FOV, $256 \times 128$ matrix, one hundred twenty $1.2-\mathrm{mm}$ thick sections, TE/TR $=1.71 / 1900 \mathrm{~ms}$ ), proton attenuation/T2-weighted $(230 \times 230 \mathrm{~mm}$ FOV, $320 \times 320 \mathrm{ma}-$ trix, forty-eight 3 -mm sections, TE1/TE2/TR $=20 / 91 / 3600 \mathrm{~ms}$ ), and T1 fast low-angle shot $(230 \times 230 \mathrm{~mm}$ FOV, $320 \times 320$ matrix, forty-eight 3 -mm sections, TE/TR $=2.46 / 300 \mathrm{~ms}$, flip angle $=75^{\circ}$ ) postgadolinium $(0.1 \mathrm{mmol} / \mathrm{kg})$. Total time of each DTI scan was 10 minutes, 40 seconds, for a total scanning time of 47 minutes, 14 sec- onds. Conventional and DTI images were assessed within a few days of acquisition for quality, and patients returned for repeat acquisition if significant artifacts (ie, spike artifacts, motion) were observed.

\section{Image Analysis}

At baseline, all gadolinium-enhancing lesions and 20 regions of interest from NABT (Fig 1) were drawn on each subject by using AFNI. ${ }^{13}$ For gadolinium-enhancing lesions, regions of interest were drawn over the gadolinium-enhancing region of the lesion on postgadolinium T1-weighted images. For NABT, regions of interest were drawn onto the FA maps to improve the accuracy of longitudinal coregistration. NABT regions of interest were chosen to represent varied white matter and deep gray matter regions. NABT regions of interest were not drawn on areas that showed abnormal signal intensity on either T1-weighted or T2-weighted images at baseline or any follow-up scan. Tissue immediately adjacent to CSF was not included in either gadolinium-enhanced lesions or NABT because of possible later coregistration errors that may include CSF. Regions of interest were followed longitudinally by coregistration of anatomic T1MPRAGE images acquired at months $1,2,6$, and 12 to the T1MPRAGE image acquired at baseline by using Functional MRI of the Brain Software Library. ${ }^{14}$ Regions of interest were inspected through AFNI at each time point to confirm the accuracy of coregistration and were adjusted when necessary. The reproducibility of these measures in NABT by using this coregistration technique was shown in a previous study, with a median coefficient of variation over 3 annual scans of $9.59 \%$ for FA, $2.21 \%$ for apparent diffusion coefficient (comparable with MD), $3.70 \%$ for $\lambda_{\|}$, and $4.19 \%$ for $\lambda_{\perp}$. Enhancing lesions at baseline were classified at 12 months as either isointense or hypointense (black holes) on T1 images. Isointensity was defined as an inability to visualize any portion of the lesion on nonenhanced $\mathrm{T} 1$ images. T1-hole designation at 12 months was then confirmed by a second investigator, and both investigators were blinded to DTI measures. Average values within each region of interest were derived for FA, $\lambda_{\perp}, \lambda_{\|}$, and MD. For descriptive purposes, QBrain Version 1.1.20.0 (Medis Medical Imaging Systems, Leiden, the Netherlands) was used to measure $\mathrm{T} 2$ lesion volumes.

\section{Statistical Analysis}

Longitudinal analysis and group comparisons were performed by using mixed-model analysis, with slope (ie, DTI change per month) as the dependent variable. The ability of baseline DTI measures within gadolinium-enhancing lesions to predict T1 black holes at 12 months was evaluated by using either a Satterthwaite or pooled $t$ test, depending on equality of variances for each DTI measure. All assumptions of each statistical model were met. Because of multiple comparisons, significance was set at $P<.01$. Statistical analysis was performed by 1 coauthor (X.W.). Because this was an exploratory study and few longitudinal DTI data are available in patients with MS, no formal power calculations were performed.

\section{Results}

A description of the 21 patients with MS is found in the Table. As a group, these patients are similar to those in previous natalizumab clinical trials (Miller, Polman, Rudick). ${ }^{32-34}$ At baseline, 11 of 21 patients demonstrated a total of 60 (median $=5$ ) enhancing lesions. At 12 months, 26 (43\%) of those enhancing lesions were T1 black holes. There was $85 \%$ compliance with natalizumab therapy. 

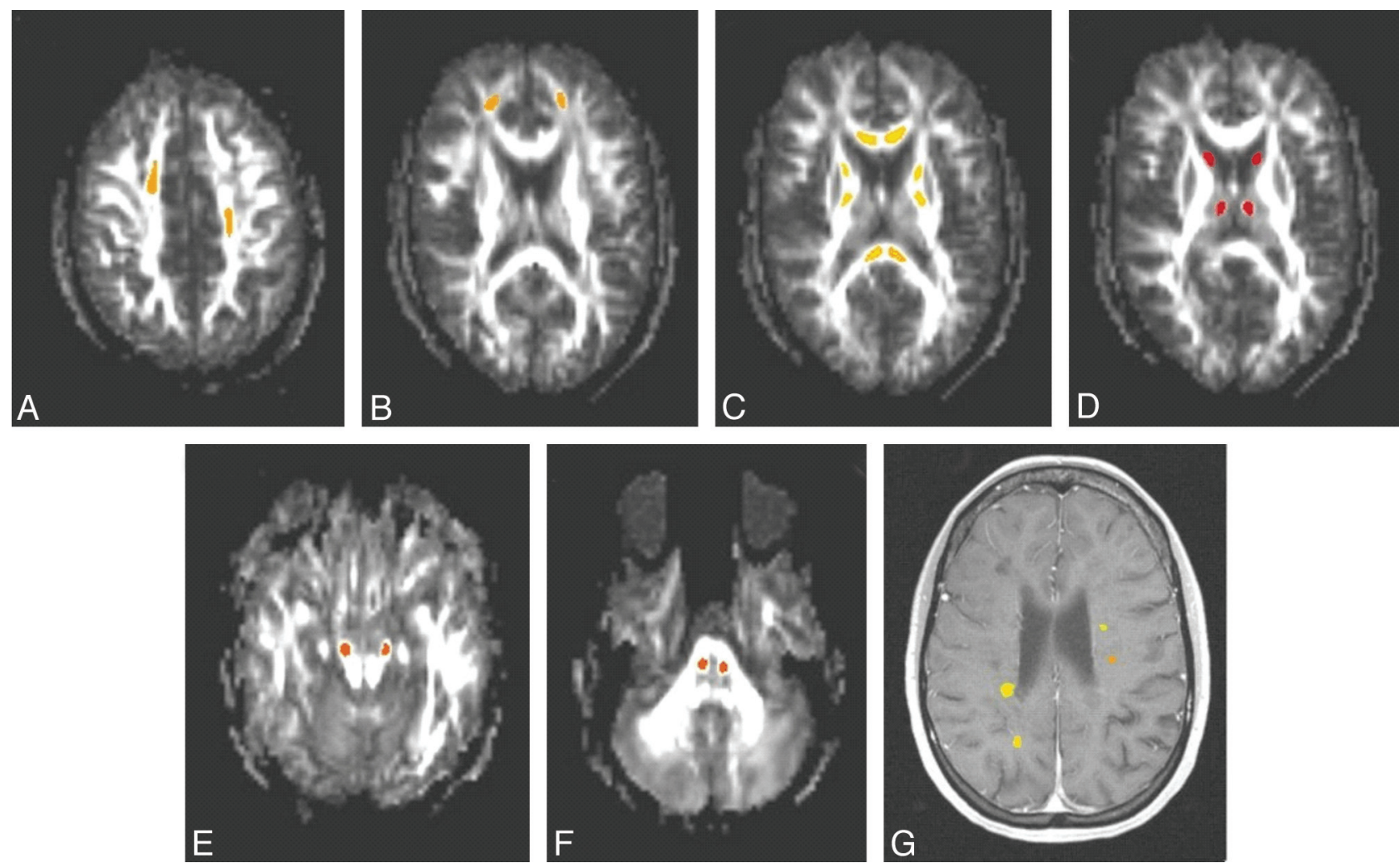

Fig 1. Sample regions of interest drawn on a single patient. NABT regions of interest are drawn on FA maps $(A-F)$, and gadolinium-enhancing lesions are drawn on a postgadolinium T1-weighted MPRAGE image $(G)$. The asymmetric placement of regions of interest in $A$ demonstrates a T2 lesion in the right centrum semiovale (T2 images not shown) in which the region of interest had to be shifted anteriorly.

\begin{tabular}{|c|c|}
\hline \multicolumn{2}{|c|}{ Description of the 21 patients with MS } \\
\hline Patient Characteristics & Data \\
\hline Age & $41.6(9.7)$ yrs \\
\hline Sex & 15 Female, 6 male \\
\hline Disease type & $\begin{array}{l}18 \text { relapsing-remitting, } 3 \text { secondary- } \\
\text { progressive (with relapses) }\end{array}$ \\
\hline Disease duration & $11.9(7.5) \mathrm{yr}$ \\
\hline Time since diagnosis & $8.0(4.6) \mathrm{yr}$ \\
\hline T2 lesion volume & $14.8(10.1) \mathrm{mL}^{3}$ \\
\hline \multicolumn{2}{|l|}{ Enhancing lesions at baseline } \\
\hline Proportion of patients & $11 / 21(52.4 \%)$ \\
\hline No. of lesions per patient & 2.9 (median, 5; range, 0-13) \\
\hline Lesion volume ( \pm SD) & $1226(1207) \mathrm{mm}^{3}$ \\
\hline \multicolumn{2}{|l|}{ NABT ROls } \\
\hline ROI volume ( $\pm S D)$ & $502.4(260.2) \mathrm{mm}^{3}$ \\
\hline
\end{tabular}

${ }^{a}$ All values are mean $( \pm S D)$ unless otherwise noted.

\section{DTI Changes Following Acute Inflammatory Injury}

During 1 year, FA increased in gadolinium-enhancing lesions ( $+2.10 /$ month, $P<.0001$; Fig 2). This increase was most prominent during the first 2 months and then became more gradual. Changes in FA within gadolinium-enhancing lesions were driven by decreased $\lambda_{\perp}\left(-1.9510^{-6} \mathrm{~mm}^{2} / \mathrm{s} / \mathrm{month}, P<\right.$ $.003)$. MD was unchanged in gadolinium-enhancing lesions $(P=$ n.s., data not shown $)$.

Higher $\lambda_{\perp}$ within gadolinium-enhancing lesions at baseline predicted conversion to T1 black holes at 12 months $(P=$ .0007). FA at baseline showed a trend for predicting T1 black holes at 12 months $(P=.011)$. Changes in DTI measures during 1 year did not differ between enhancing lesions that be- came T1 black holes and lesions that did not become T1 black holes (Fig 3).

\section{DTI Changes in NABT}

During 1 year, FA decreased in NABT $(-1.02 /$ month, $P<$ .0001 ; Fig 4). This decrease was gradual during the entire 12-month follow-up. Changes in FA within NABT were driven by decreased $\lambda_{\|}\left(-1.9210^{-6} \mathrm{~mm}^{2} / \mathrm{s} /\right.$ month, $\left.P<.0001\right)$. MD was unchanged in $\operatorname{NABT}(P=$ n.s., data not shown $)$.

\section{Discussion}

We used longitudinal evaluation of patients with MS starting anti-inflammatory therapy to evaluate the ability of DTI to characterize changes within areas of focal inflammatory injury. Within gadolinium-enhancing lesions, we observed increased FA and decreased $\lambda_{\perp}$ during the first 2 months. Remyelination is well-known to occur following acute demyelination injury, ${ }^{15-17}$ and the reduction in $\lambda_{\perp}$ may represent an imaging correlate of remyelination. Experimental animal studies have suggested that $\lambda_{\perp}$ is a somewhat specific measure of myelin integrity. Demyelination without axonal injury is induced in cuprizone-treated mice, and changes in $\lambda_{\perp}$ paralleled pathologically confirmed demyelination and remyelination. ${ }^{18}$ DTI studies in shiverer mice (who have congenital dysmyelination with intact axons) found increased $\lambda_{\perp}$ but normal $\lambda_{\|} \cdot{ }^{9}$ In the EAE mouse model, focal regions of myelin loss are associated with decreased $\lambda_{\perp} \cdot{ }^{4}$ Animal ischemia studies also found increased $\lambda_{\perp}$ but no change $\lambda_{\|}$, and histopathology showed reduced myelination. ${ }^{19}$ DTI studies from a mouse traumatic brain injury model found reduced FA 

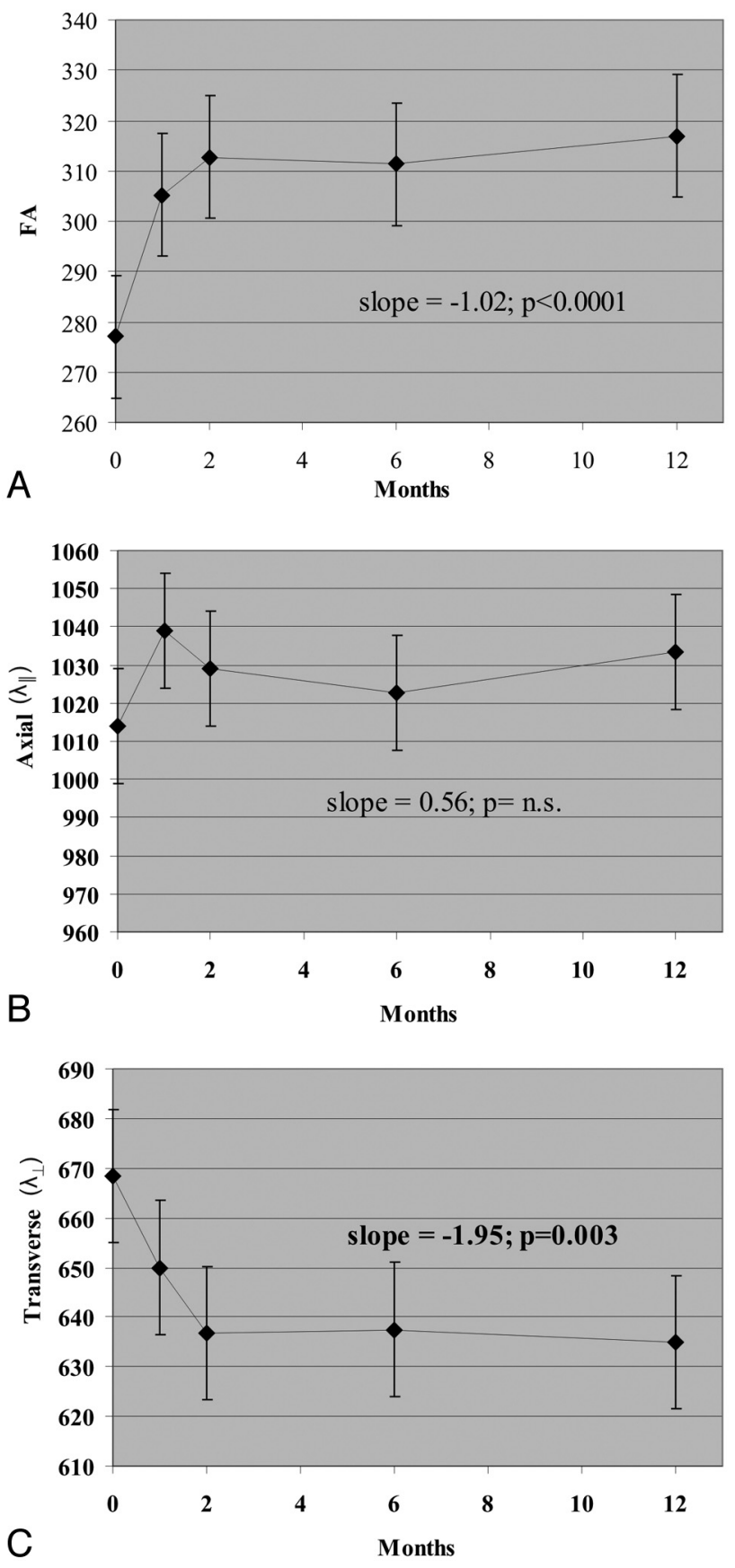

Fig 2. Change in DTI measures within gadolinium-enhancing lesions during 1 year. $A$, FA. $B, \lambda_{\|} . C, \lambda_{\perp}$. FA is unitless $\left(10^{-3}\right)$; axial and radial diffusivities are $10^{-6} \mathrm{~mm}^{2} / \mathrm{s}$.

and $\lambda_{\|}$, which corresponded to increased staining for amyloid precursor protein and neurofilament, both indicators of axonal injury. ${ }^{20}$ Altogether, these animal imaging studies suggest that the changes in $\lambda_{\perp}$ we observed within gadoliniumenhancing lesions possibly represent remyelination.

An alternative pathologic explanation for the longitudinal increased FA within gadolinium-enhancing lesions is resolution of edema and inflammatory infiltrates. However, DTI studies from other cerebral edema models suggest that there is little contribution of resolving edema to our DTI observations. Cerebral edema from hepatic encephalopathy is associated with increased MD and variable changes in FA. ${ }^{21,22}$ After lactulose therapy for hepatic encephalopathy, MD de-
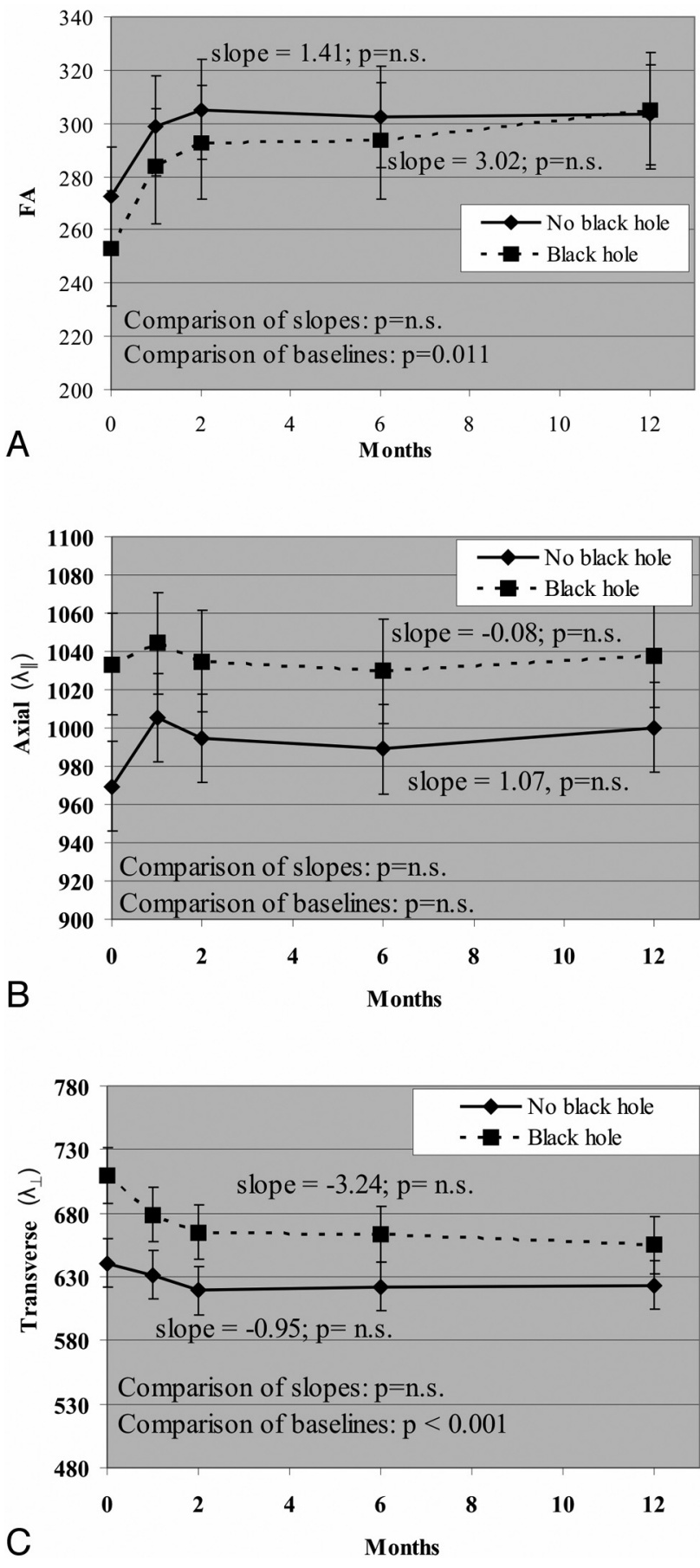

Fig 3. Change in DTI measures between gadolinium-enhancing lesions that evolved into T1 holes by 12 months and gadolinium-enhancing lesions that did not evolve into T1 black holes by 12 months. $A$, FA. $B, \lambda_{\|} . C, \lambda_{\perp}$. No-black-hole-versus-black-hole comparison refers to comparison slopes. FA is unitless $\left(10^{-3}\right)$; axial and radial diffusivities are $10^{-6} \mathrm{~mm}^{2} / \mathrm{s}$.

creased while FA did not change. ${ }^{22}$ Peritumoral edema shows both a decrease in FA and an increase in apparent diffusion coefficient (which is comparable with MD). ${ }^{23,24}$ Individual eigenvalue analysis of peritumoral edema showed increase in both $\lambda_{\perp}$ and $\lambda_{\|}$. In contrast, we observed no change in either MD or $\lambda_{\|}$. Furthermore, we observed a change in $\lambda_{\perp}$ alone, with no change in $\lambda_{\|}$. Although the resolution of gadoliniumenhancing lesions likely involves some resolving edema, comparison of our DTI observations with those from hepatic en- 

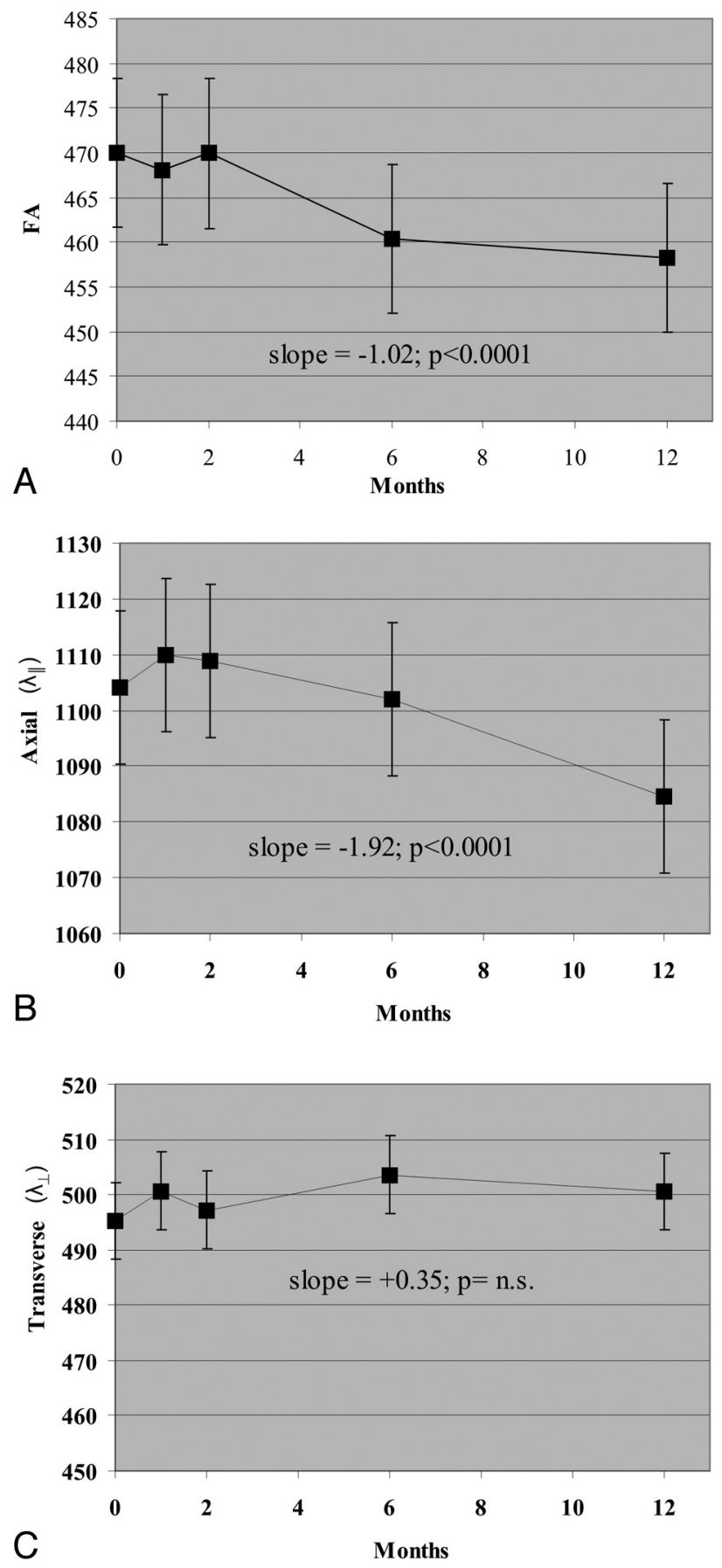

Fig 4. Change in DTI measures within normal-appearing brain regions of interest during 1 year. $A, F A . B, \lambda_{\|}$. C, $\lambda_{\perp}$. FA is unitless $\left(10^{-3}\right) ; \lambda_{\|}$and $\lambda_{\perp}$ are $10^{-6} \mathrm{~mm}^{2} / \mathrm{s}$.

cephalopathy and cerebral tumors suggest that this represents only a small proportion of tissue recovery.

Chronic T1 hypointensities (T1 black holes) are associated with severe tissue damage, as measured by axonal loss, axonal swelling, and demyelination..$^{25-27}$ To date, no published studies have evaluated DTI predictors of conversion from gadolinium-enhancing lesions to T1 black holes. In our study, approximately half of the gadolinium-enhancing lesions developed into T1 black holes by 12 months. $\lambda_{\perp}$ within gadolinium-enhancing lesions at baseline predicted evolution into $\mathrm{T} 1$ black holes, and a trend was observed with FA. In contrast, we observed no difference in change in DTI measures during 1 year of follow-up between gadolinium-enhancing lesions that became T1 black holes and those that did not. These observations suggest that evolution of gadolinium-enhancing lesions into T1 black holes is influenced predominantly by the degree of initial injury and not the amount of later recovery. These observations also add to the accumulating evidence that DTI is a quantitative measure of the degree of brain tissue injury.

We also applied DTI to study changes in NABT with time during MS therapy. This longitudinal analysis showed gradual decline in FA during 1 year of follow-up. In contrast to enhancing lesions, this decline was driven by a decrease in $\lambda_{\|}$, with no significant change in $\lambda_{\perp}$. Animal studies of inflammatory injury found a relative specificity of $\lambda_{\|}$to represent axonal injury. DTI studies of the spinal cord of mice with EAE showed decreased $\lambda_{\perp}$ throughout the cord, which correlated with increased staining of antibodies against the axonalrelevant $\beta$-amyloid precursor protein. ${ }^{4}$ Retinal ischemia causes wallerian degeneration of the optic nerve, leaving myelin intact for a period of time. Three days after induction of retinal ischemia, DTI of the optic nerve shows decreased $\lambda_{\|}$ with no change in $\lambda_{\perp}{ }^{8}$ Subsequent pathology confirmed axonal degeneration without demyelination. A study of patients with corpus callosotomy found an early decrease in $\lambda_{\|}$in the white matter adjacent to the surgery, which was followed by a later decline in $\lambda_{\perp} \cdot{ }^{6}$ This imaging time course parallels the wallerian degeneration following axonotomy, with distal axonal loss first, followed later by myelin loss.

These animal studies suggest that our observed decline in $\lambda_{\|}$represents an ongoing degenerative process outside of demyelinating lesions, which preferentially affects axonal integrity. The changes we observed continued despite antiinflammatory therapy. Continued degeneration in NABT was also observed as accelerated brain atrophy in natalizumabtreated patients compared with healthy controls. ${ }^{28}$ Our studies suggest that a similar process driven by axonal loss may be occurring in the NABT in patients with MS, even in the absence of active inflammation as measured by clinical relapses and gadolinium-enhancing lesions. There was no change with time in MD within NABT, which suggests that changes in FA and $\lambda_{\|}$were not secondary to resolution of generalized edema.

There are few other longitudinal DTI studies in MS, and no studies have evaluated patients after starting a specific therapy. One 18-month study of untreated patients with MS found increased MD and decreased FA with time in chronic MS lesions. MD also increased in normal-appearing gray matter, but not NAWM. ${ }^{29}$ Another study of mostly patients with progressive MS found that MD (but not FA) increased during 2.4 years in NAWM. ${ }^{30}$ We studied patients at an earlier stage of disease and after starting anti-inflammatory therapy, so our observations more accurately characterize a stage of disease that has greater potential for tissue recovery. In addition, we were able to characterize the continued neurodegeneration in NABT, despite cessation of active inflammation. Finally, compared with our study using 71 diffusion gradients, both previous DTI studies used an 8 -direction pulse sequence. It has been shown that at least 20 and 30 directions are necessary for robust determination of anisotropy and mean diffusivity, respectively. ${ }^{31}$ 
In an open-label study with no formal control group, it may be difficult for our data to differentiate the effect of antiinflammatory therapy from the natural course of MS. Gadolinium enhancement always resolves, so the increase in FA may be secondary simply to the natural resolution of inflammation in this disease. DTI measures vary from 1 brain region to another and from 1 patient to another, making comparison studies difficult to interpret. Our longitudinal comparison of the same brain regions within the same patients minimized this problem. Furthermore, there is no pathologic data comparing treated and untreated patients with MS, making it difficult to extrapolate imaging data to pathologic changes. Future studies comparing treated and untreated patients are needed to identify the effects of MS therapy on DTI measures of tissue integrity more clearly. This study enrolled patients with MS with variable disease duration and different prior MS therapies, irrespective of other comorbid conditions. Although this process introduces variability in the dataset, it also allows greater generalization of this dataset to general clinical practice and future clinical trials. Indeed, the subjects are representative of both the general MS population and those studied in the major natalizumab clinical trials, making these observations widely applicable to the general MS patient population.

It is well-established that natalizumab therapy reduces new lesions as measured by conventional imaging, ${ }^{32-34}$ but less known is the impact of any immunosuppressive therapy on advanced imaging measures. The rate of progressive brain atrophy is slower in patients treated with natalizumab, but only in the second year of therapy. ${ }^{28}$ Natalizumab therapy was associated with a reduction in conversion of gadoliniumenhancing lesions into T1 black holes. ${ }^{35}$ No studies have evaluated the potential impact of natalizumab therapy on advanced imaging measures, such as DTI. Natalizumab prevents leukocyte adhesion to the capillary endothelial surface, thereby reducing leukocyte transmigration into the central nervous system. ${ }^{36,37}$ Natalizumab is associated with a rapid and robust reduction in the development of new gadoliniumenhancing lesions, ${ }^{32}$ which is expected to create a less inflammatory environment, thus allowing more effective repair. Furthermore, enhancing lesions in patients started on natalizumab are less likely to later convert to T1 black holes. ${ }^{35}$ These observations suggest that natalizumab therapy may have a positive impact on tissue integrity, even in regions with active inflammation.

Our observation suggests that during treatment with natalizumab, there is tissue recovery in areas of active inflammation, as measured by increased FA and reduced $\lambda_{\perp}$. Perhaps more important, we observed continued decline in FA and $\lambda_{\|}$ within NABT, despite treatment with a highly effective antiinflammatory therapy. These observations add further evidence to the pathophysiologic separation between inflammation and degeneration. ${ }^{38,39}$ Additional studies are needed to compare our observations in natalizumab-treated patients with MS with untreated patients with MS.

Our results imply that DTI may provide pathology-specific insights into MS, because FA was driven by different eigenvectors in gadolinium enhancing lesions and NABT. If axial and radial diffusion indeed are relatively specific for axonal injury and demyelination, respectively, then DTI may be a useful biomarker of tissue recovery and later degeneration. Additionally, this study demonstrates that DTI is a dynamic measure of tissue integrity, with the ability to measure both short-term tissue recovery and long-term degeneration. These observations suggest that DTI may be a useful metric of therapeutic trials of tissue recovery and antidegeneration. These studies should target FA and individual diffusivities $\left(\lambda_{\perp}\right.$ for tissue recovery and $\lambda_{\|}$for antidegeneration) instead of MD, because these appear to be more dynamic metrics with time.

\section{Conclusions}

We observed changes in DTI measures during 1 year in both gadolinium-enhancing lesions and NABT. The DTI results may represent possible remyelination within acute lesions and chronic axonal degeneration in NAWM. Evolution of gadolinium-enhancing lesions into T1 black holes is related more to the degree of initial injury than the degree of later recovery. These results support the use of DTI as a measure of tissue integrity for studies of neuroprotective therapies.

\section{References}

1. Fox RJ. Picturing multiple sclerosis: conventional and diffusion tensor imaging. Semin Neurol 2008;28:453-66

2. Basser PJ, Pierpaoli C. Microstructural and physiological features of tissues elucidated by quantitative-diffusion-tensor MRI. J Magn Reson 1996;111: 209-19

3. Pierpaoli C, Basser PJ. Toward a quantitative assessment of diffusion anisotropy. Magn Reson Med 1996;36:893-906

4. Kim JH, Budde MD, Liang HF, et al. Detecting axon damage in spinal cord from a mouse model of multiple sclerosis. Neurobiol Dis 2006;21:626-32

5. DeBoy CA, Zhang J, Dike S, et al. High-resolution diffusion tensor imaging of axonal damage in focal inflammatory and demyelinating lesions in rat spinal cord. Brain 2007;130:2199-210

6. Concha L, Gross DW, Wheatley BM, et al. Diffusion tensor imaging of timedependent axonal and myelin degradation after corpus callosotomy in epilepsy patients. Neuroimage 2006;32:1090-99

7. Schmierer K, Wheeler-Kingshott CA, Boulby PA, et al. Diffusion tensor imaging of post mortem multiple sclerosis brain. Neuroimage 2007;35:467-77. Epub 2006 Dec 16

8. Song SK, Sun SW, Ju WK, et al. Diffusion tensor imaging detects and differentiates axon and myelin degeneration in mouse optic nerve after retinal ischemia. Neuroimage 2003;20:1714-22

9. Song SK, Sun SW, Ramsbottom MJ, et al. Dysmyelination revealed through MRI as increased radial (but unchanged axial) diffusion of water. Neuroimage 2002;17:1429-36

10. Fox RJ, McColl RW, Lee JC, et al. A preliminary validation study of diffusion tensor imaging as a measure of functional brain injury. Arch Neurol 2008; 65:1179-84

11. Lowe MJ, Horenstein C, Hirsch JG, et al. Functional pathway-defined MRI diffusion measures reveal increased transverse diffusivity of water in multiple sclerosis. Neuroimage 2006;32:1127-33

12. Naismith RT, Xu J, Tutlam NT, et al. Disability in optic neuritis correlates with diffusion tensor-derived directional diffusivities. Neurology 2009;72:589-94

13. Cox RW. AFNI: software for analysis and visualization of functional magnetic resonance neuroimages. Comput Biomed Res 1996;29:162-73

14. Smith SM, Jenkinson M, Woolrich MW, et al. Advances in functional and structural MR image analysis and implementation as FSL. Neuroimage 2004; 23(suppl 1):S208-19

15. Prineas JW, Barnard RO, Kwon EE, et al. Multiple sclerosis: remyelination of nascent lesions. Ann Neurol 1993;33:137-51

16. Raine CS, Wu E. Multiple sclerosis: remyelination in acute lesions. J Neuropathol Exp Neurol 1993;52:199-204

17. Prineas JW, Kwon EE, Cho ES, et al. Continual breakdown and regeneration of myelin in progressive multiple sclerosis plaques. Ann N Y Acad Sci 1984; 436:11-32

18. Song SK, Yoshino J, Le TQ, et al. Demyelination increases radial diffusivity in corpus callosum of mouse brain. Neuroimage 2005;26:132-40

19. Wang S, Wu EX, Tam CN, et al. Characterization of white matter injury in a hypoxic-ischemic neonatal rat model by diffusion tensor MRI. Stroke 2008;39:2348-53

20. Mac Donald CL, Dikranian K, Song SK, et al. Detection of traumatic axona injury with diffusion tensor imaging in a mouse model of traumatic brain injury. Exp Neurol 2007;205:116-31 
21. Kumar R, Gupta RK, Elderkin-Thompson V, et al. Voxel-based diffusion tensor magnetic resonance imaging evaluation of low-grade hepatic encephalopathy. J Magn Reson Imaging 2008;27:1061-68

22. Kale RA, Gupta RK, Saraswat VA, et al. Demonstration of interstitial cerebral edema with diffusion tensor MR imaging in type $\mathrm{C}$ hepatic encephalopathy. Hepatology 2006;43:698-706

23. Toh $\mathrm{CH}$, Castillo M, Wong AM, et al. Differentiation between classic and atypical meningiomas with use of diffusion tensor imaging. AJNR Am J Neuroradiol 2008;29:1630-35

24. Sundgren PC, Fan X, Weybright P, et al. Differentiation of recurrent brain tumor versus radiation injury using diffusion tensor imaging in patients with new contrast-enhancing lesions. Magn Reson Imaging 2006;24:1131-42

25. van Walderveen MA, Kamphorst W, Scheltens P, et al. Histopathologic correlate of hypointense lesions on T1-weighted spin-echo MRI in multiple sclerosis. Neurology 1998;50:1282-88

26. Bitsch A, Kuhlmann T, Stadelmann C, et al. A longitudinal MRI study of histopathologically defined hypointense multiple sclerosis lesions. Ann Neurol 2001;49:793-96

27. Fisher E, Chang A, Fox RJ, et al. Imaging correlates of axonal swelling in chronic multiple sclerosis brains. Ann Neurol 2007;62:219-28

28. Miller DH, Soon D, Fernando KT, et al. MRI outcomes in a placebo-controlled trial of natalizumab in relapsing MS. Neurology 2007;68:1390-401

29. Oreja-Guevara C, Rovaris M, Iannucci G, et al. Progressive gray matter damage in patients with relapsing-remitting multiple sclerosis: a longitudinal diffusion tensor magnetic resonance imaging study. Arch Neurol 2005;62:578-84

30. Agosta F, Absinta M, Sormani MP, et al. In vivo assessment of cervical cord damage in MS patients: a longitudinal diffusion tensor MRI study. Brain 2007;130:2211-19

31. Jones DK. The effect of gradient sampling schemes on measures derived from diffusion tensor MRI: a Monte Carlo study. Magn Reson Med 2004; 51:807-15

32. Miller DH, Khan OA, Sheremata WA, et al. A controlled trial of natalizumab for relapsing multiple sclerosis. New Engl J Med 2003;348:15-23

33. Rudick RA, Stuart WH, Calabresi PA, et al. Natalizumab plus interferon beta-1a for relapsing multiple sclerosis. $N$ Engl J Med 2006;354:911-23

34. Polman C, O'Connor P, Havrdova E, et al. Clinical results from AFFIRM: a randomized double-blind, placebo-controlled, multicentrer trial to determine the efficacy and safety of natalizumab in patients with relapsing multiple sclerosis (MS). Neurology 2005;64(suppl 1):A146

35. Dalton CM, Miszkiel KA, Barker GJ, et al. Effect of natalizumab on conversion of gadolinium-enhancing lesions to $\mathrm{T} 1$ hypointense lesions in relapsing multiple sclerosis. J Neurol 2004;251:407-13

36. Brocke S, Piercy C, Steinman L, et al. Antibodies to CD44 and integrin alpha4, but not L-selectin, prevent central nervous system inflammation and experimental encephalomyelitis by blocking secondary leukocyte recruitment. Proc Natl Acad Sci U S A 1999;96:6896-901

37. Yednock TA, Cannon C, Fritz LC, et al. Prevention of experimental autoimmune encephalomyelitis by antibodies against alpha 4 beta 1 integrin. Nature 1992;356:63-66

38. Trapp BD, Nave KA. Multiple sclerosis: an immune or neurodegenerative disorder? Annu Rev Neurosci 2008;31:247-69

39. Imitola J, Chitnis T, Khoury SJ. Insights into the molecular pathogenesis of progression in multiple sclerosis: potential implications for future therapies. Arch Neurol 2006;63:25-33 